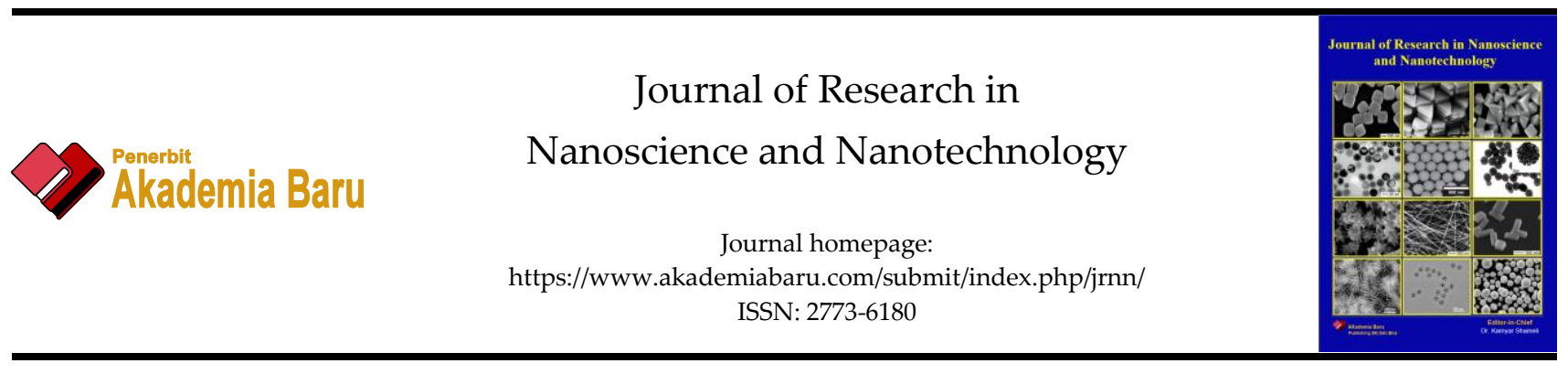

\title{
Biosynthesis of Silver Nanoparticles Using Juglans Regia Green Husk (Walnut) Water Extract and evaluation Antibacterial activity
}

\section{Zahra Izadiyan 1,*, Kamyar Shameli 1,* , Zahra Maghareh Esfahan ${ }^{2}$, Siti Husnaa Mohd Taib ${ }^{\text {, }}$ and Pooneh Kia ${ }^{3}$}
${ }^{1}$ Department of Environment and Green Technology, Malaysia-Japan International Institute of Technology, Universiti Teknologi Malaysia, Jalan Sultan Yahya Petra, 54100 Kuala Lumpur, Malaysia
${ }^{2}$ Department of Chemical \& Environmental Engineering, Faculty of Engineering, Universiti Putra Malaysia, 43400, Serdang, Selangor, Malaysia
${ }^{3}$ Institute of Bioscience, Universiti Putra Malaysia, 43400 UPM Serdang, Selangor, Malaysia
* Correspondence: Kamyar Shameli, E-mail: kamyarshameli@gmail.com; Tel.: +603-22031200, Zahra Izadiyan, Email: Zahra_izadiyan@yahoo.com.my https://doi.org/10.37934/jrnn.3.1.90102

\begin{abstract}
Biosynthesis of Ag-NPs at room temperature by using Juglans regia (J. regia) green husk extract which acts as reductant and stabilizer, simultaneously. The Ag/J. regia were characterized by using UV-visible, zeta potential, TEM, and AFM. Formation of Ag/ J. regia was determined by UV-vis spectroscopy, where absorption maxima surface plasmon at 400-460 $\mathrm{nm}$. The zeta potential analysis indicated that J. regia green husk extract was negative and increasing in Ag/ J. regia. TEM images show the mean particle size was 31.37 $\mathrm{nm}$ with the standard deviation of $7.1 \mathrm{~nm}$, where confirm by AFM measurements. The XRD study indicates the crystalline nature of the Ag-NPs. The antibacterial activity of Ag-NPs was investigated against Grampositive and Gram-negative bacteria by the disc diffusion method were found to have high antibacterial activity. These results show that Ag-NPs can be useful in different biologic research and biomedical applications.
\end{abstract}

Keywords:

Silver nanoparticles, biosynthesis, Juglan

sregia, green husk, antimicrobial activity.

\section{Introduction}

Researchers in the past decade have been paying great attention to the field of nanotechnology that involves reactions at molecular as well as atomic levels. Nanotechnology includes the characterization, synthesis, as well as device and material application of the tiniest parts that measure 5 at least a dimension on scales of $100 \mathrm{~nm}$ and lower [1]. The field of nanotechnology provides a wide 
range of technological foundation for applications in various areas including antibacterial [2], biosensor [3] industrial bio-processing as well as molecular medicine[4] and biomedical applications [5].

Recently, nanotechnology, particularly metal nanoparticles, have emerged as a rapidly new field due to their unique chemical and physical properties in technological innovations and industry [6]. Synthesis of nanoparticles using green methods has drawn a growing interest due to their environmentally friendly and low-cost aspects compared with current chemical and physical methods [7].

Nanoparticles that are metallic are particularly in demand in the engineering and biomedical science fields due to their great potential in the area of nanotechnology, thereby leading to a large amount of potential application in the separation of magnetic and biotechnology field [8].In the synthesis of silver nanoparticles (Ag-NPs) via green method, a solution of silver salt is reduced using anextract of the plant. This process involves a chemical reaction which takes place through two steps: 1. formation of small silver atoms nuclei as nucleation phase, 2. and growth phase containing grouping these small nuclei, in which leads to nanoparticles creation [9].

Silver ions can be reduced using several methods such as by using $\gamma$-rays[10], ultraviolet (UV) irradiation [11], reduction of electrochemical and heating[12], as well as by applying decreasing chemicals, involving sodium borohydride [13], hydrazine [14], N,N-dimethylformamide [15], polyethylene glycerol [16], glucose [17], formaldehyde [18], ethylene glycol [19], and sodium in ammonium liquid [20]. Nevertheless, further economic, financially suitable, and environmentally friendly synthesis path in synthesizing Ag-NPs is required. Ag-NPs' green synthesis includes three major phases that should be assessed according to the perspectives of green chemistry such as selecting the medium for solvent, agent for reducing, as well as a stabilizer that is non-toxic on AgNPs [21].

The nanoparticles' biosynthesis that involves the link between biotech and nanotech, has been getting growing attention given the increasing requirement for developing technologies that are environmentally friendly for the synthesis of materials. Search for the best biomaterial for nanoparticles' biosynthesis is continued via various methods that are synthetic. The method of biosynthetic utilizing extracts from plants has gained more interest compared to methods using chemicals and physical approaches including the utilization of microbes. The approach is appropriate for the metal synthesis at a nanoscale given the lack of any need for maintaining an environment that is aseptic [22].

Different parts of the walnut tree (Juglans regia) such as kernels, leaves, tree bark and also fruit green huskwere utilized for both industries of pharmaceuticals and cosmetics [23]. The study by Carvalho et al. (2010) established the activity of the antioxidant in walnut leaves, seeds, and green husks, as well as in antimicrobial activities [24]. The J. regia aqueous extracts were examined by Ghasemiet al. (2011) studied the methanolic ones [25].

The findings of Carvalhoet al. (2010) display the potential of these low-cost natural materials as the source of compounds that are phenolic with activities of antimicrobial and antiradical and it also reveals that green husk knowledge should be widened [26].

Based on past literature review, the phenols' content varies from the J. regia. The highperformance liquid chromatography approach utilized in determining the external standards has allowed the act of identifying six compounds that are phenolic including vanillic acid, myricetin, coumaric acid, syringic acid, juglone, and ferulic acid [27-28]. All the above results are matching with phenols as represented in Figure 1. According to this schematic illustration that we suggest, they could beinvolved closely in the reducing and stabilizing of $\mathrm{Ag}+$ to $\mathrm{Ag}^{\circ}$ where the presence of electrons from oxygen atoms helped in the absorption of compounds on Ag-NPs [29]. 
In the present work, the walnuts extract was proposed to be a suitable and convenient plant to the green synthesis of Ag-NPs. The method used in this research is totally green and involves an easy single step process by combining the solution of $\mathrm{AgNO}_{3}$ and walnuts aqueous extract. The silver nitrate is utilized as the silver precursor and walnuts extract due to the high contents of polyphenols compounds were utilized as the stabilizer and reducing agents, simultaneous. Furthermore, utilizing nontoxic and cheap compounds in the plant extract and reaction in moderate temperature are some of these procedures advantages. As far as the researchers are aware, this is the first time that walnut extracts are used in the Ag-NPs' synthesis.

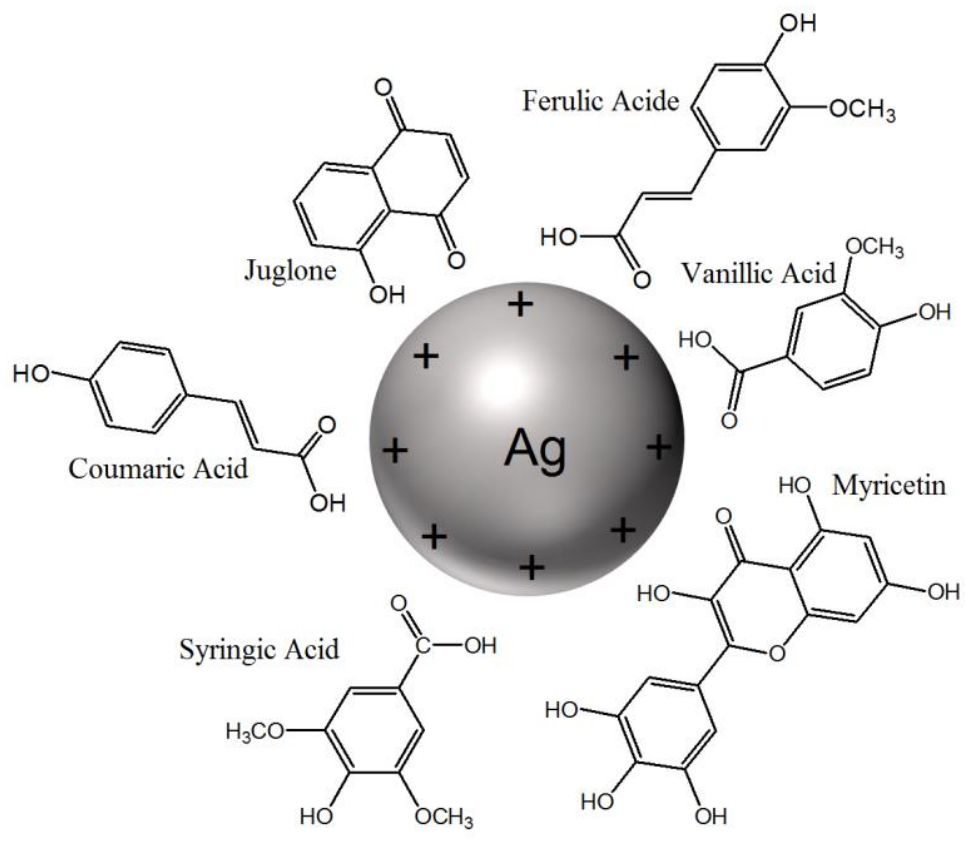

Figure 1. Schematic of synthesized Ag-NPs interactions with activated functional groups of J. regia.

\section{Materials and Methods}

\subsection{Material}

The walnut green husk was collected from Sorkh-e-Hesar Tehran, Iran and specimen of this plant identified by Iranian Research Institute of Plant Protection (IRIPP). Silver nitrate was used as a silver precursor and provided by Bendosen Company $(99.89 \%$, C0721-2284551). Nutrient agar and nutrient broth were purchased from MERCK KGaA. All reagents in this effort were analytical grade and were used as received without further purification. All solutions were freshly prepared using double distilled water and kept in the dark to avoid any photochemical reactions. All glassware used in experimental procedures were cleaned in a fresh solution of $\mathrm{HNO}_{3} / \mathrm{HCl}(3: 1, \mathrm{v} / \mathrm{v})$, washed thoroughly with double distilled water, and dried before use.

\subsection{Extraction Preparation}

Walnut green husk (Fig. 2a) was washed and dried in an oven dryer at $40{ }^{\circ} \mathrm{C}$ for $48 \mathrm{~h}$. The green husk of J. regia dry ground in a mill, stored in glass bottles and kept at room temperature for next analysis (Fig. 2b). The finely ground J. regia green husk $(0.5 \mathrm{~g})$ was added to the $100 \mathrm{ml}$ of the boiling water for $30 \mathrm{~min}$. It then was filtered through the filter paper. The concentrated extracts were kept in a dark place at $4{ }^{\circ} \mathrm{C}$ for future use (Fig. 2c). 

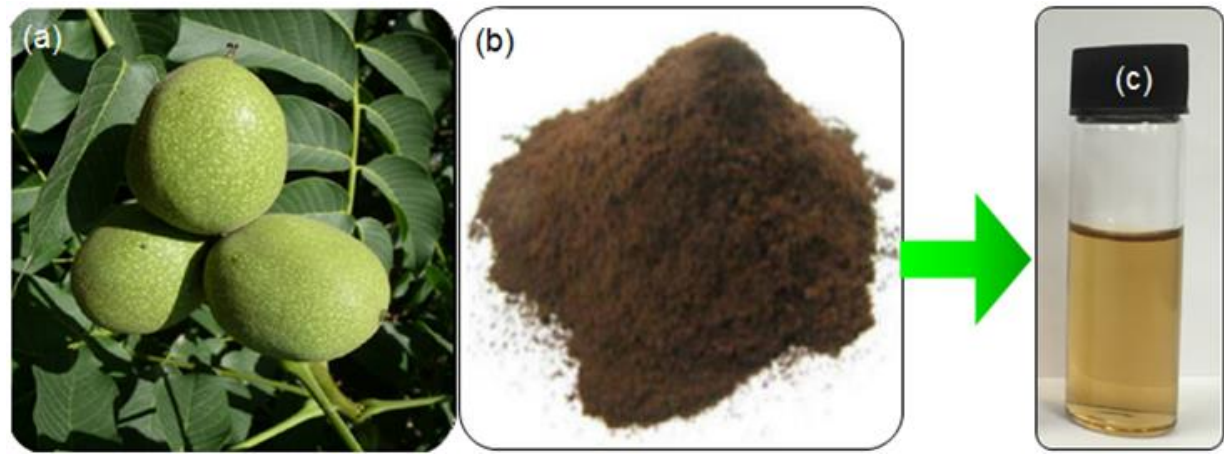

Figure 2. Juglans regia green husk (a), powder of J. regia green husk (b) and aqueous extract of J. regia green husk (c).

\subsection{Synthesis of J. regial Ag-NPs}

In a typical reaction procedure, $0.5 \mathrm{~g}$ extract of $J$. regia was added to $100 \mathrm{ml}$ distilled deionized water with vigorous stirring for $30 \mathrm{~min}$. A twenty-fivemilliliters of $\mathrm{AgNO}_{3}(5 \times 10-3 \mathrm{M})$ was added and homogenized by using a magnetic stirrer at room temperature $\left(25^{\circ} \mathrm{C}\right)$ for $10 \mathrm{~h}$. The Ag-NPs were gradually obtained during the incubation period.

\subsection{Antibacterial activity}

The in-vitro antibacterial activity of the new compounds was evaluated against two pathogenic Gram negative bacteria; E. coli (Escherichia coli), P.aeruginosa (Pseudomonas aeruginosa), and two Gram-positive bacteria, S. aureus (Staphylococcus aureus), and B. cereus (Bacillus cereus) via a disc diffusion method. The in-vitro antibacterial test was carried out in keeping with the recommended standards of the National Committee for Clinical Laboratory Standards based on the determination of the inhibition zone in millimeters $(\mathrm{mm})$ in nutrient agar, $[38,39]$. The microbe cultures were standardized to the $0.5 \mathrm{McF}$ arland standard which is approximately 108 cells. Ampicillin was applied as a positive control (10 $\mu \mathrm{g} \mathrm{mL-1} \mathrm{concentrations).} \mathrm{Briefly,} \mathrm{test} \mathrm{compounds} \mathrm{previously} \mathrm{sterilized} \mathrm{with}$ UV were inoculated with $6 \mathrm{~mm}$ diameter paper discs and then positioned on the nutrient agar surface of the microbial growth plate. The plates were inverted and incubated at $37{ }^{\circ} \mathrm{C}$ for $18-24 \mathrm{~h}$ until sufficient growth was achieved. After incubation, the diameters of the zones around the specimens showing the inhibition amount were measured in millimeters from the back of the petri plates by the ruler (naked eye).

\subsection{Characterization of the J. regial Ag-NPs}

The XRD analysis was carried out to determine the phase crystallinity and purity of the synthesized Ag-NPs (studied by using PXRD in the small angle range of 2॰ (10-90 degrees). The scan speed of 2 degrees/minutes was applied to PXRD patterns recording). The Ag/J.regia preparations were characterized by the use of ultraviolet-visible (UV 1800, SHIMADZU) spectroscopy (UV-vis) in the range $400-800 \mathrm{~nm}$. The image of TEM were characterized by applied a Hitachi H-71001 electron microscope (Hitachi High-Technologies Corporation, Tokyo, Japan), Japan transmission electron microscope (TEM). Zeta potential using the Zeta/Nano Particle Analyser, (Systems Nano-Plus, Japan), was utilized to measurement the charge of the droplet surface of solution which may cause 
effects on the chemical and physical stability of the Ag-NPs[30]. Surface roughness measured by Atomic Force Microscopy (AFM, BRUKER: Innova USA) in noncontact mode as well as provides high-resolution two-dimensional and three-dimensional image information. Surface roughness determined by AFM in tapping mode. The tapping mode is that the probe does not touch the sample during scanning but oscillates above it.

\section{Results and Discussion}

Figure $3 \mathrm{a}$ and $\mathrm{b}$ represent the reduction of $\mathrm{Ag}^{+}$into $\mathrm{Ag}-\mathrm{NPs}$ during exposure to J. regia green husk extracts could be followed by the color change. The fresh suspension of J. regia green husk was pale yellow in color. However, after addition of $\mathrm{AgNO}_{3}$ and stirring for $10 \mathrm{~h}$ at room temperature, the emulsion turned to dark brownish color. The color changes in aqueous solutions are due to the surface plasmon resonance phenomenon. The result obtained in this investigation is interesting because it can serve as a foundation in terms of identification of potential forest plants for synthesizing Ag-NPs. The color change has evidenced the reduction of $\mathrm{Ag}^{+}$ions to $\mathrm{Ag}^{\circ}$ by the green husk of $J$. regia extraction via aredox reaction. Inspection of the sample by UV-vis revealed that the optimum point after $10 \mathrm{~h}$ to the reaction at room temperature was obtained.

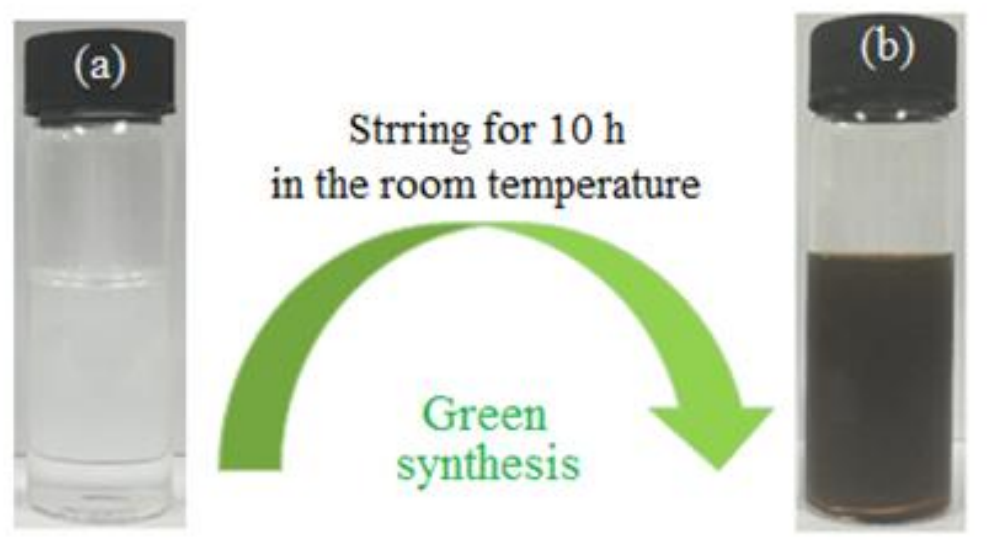

\section{Silver ions $(0,005 \mathrm{M})+$ Extract of $J$. regia green husk}

Figure 3. Photograph of color changing during of reducing Silver nitrate (a) to Ag-NPs after $10 \mathrm{~h}$.

Juglans regia green husk as a source of carbonyl and phenolic groups can reduce silver ions to Ag-

NPs. The possible chemical equations for preparing the Ag-NPs are:

$21 \mathrm{Ag}_{(\mathrm{aq})}^{+}+$J. regia $\stackrel{\text { stirring at the room temperature }}{\longrightarrow}[\mathrm{Ag} / J \text {. regia }]^{+}$

$22[\mathrm{Ag} / J \text {. regia }]^{+} \stackrel{\text { stirringfor } 10 h}{\longrightarrow}[\mathrm{Ag} / J$. regia $] \downarrow$

After dispersion of silver ions in the J. regia aqueous solution matrix (Equation 1), the extract was reacted with the $\mathrm{Ag}$ to form $[\mathrm{Ag} / J \text {. regia }]^{+}$complex, which reacted with functional groups in the molecular structure to form $[\mathrm{Ag} / J$. regia $]$ due to the reduction of silver ions through the oxidation process (Equation 2). 


\subsection{X-Ray Diffraction Analysis}

The crystallinity of J. regia/Ag-NPs has been studied by the XRD pattern. Figure 3 shows the XRD pattern of J. regia and the J. regia/Ag-NPs. Figure 4(a) indicates the peak at 28.81which corresponded to the J. regia structure. In addition, Figures 4 (b) shows the J. regia/Ag-NPs after $10 \mathrm{~h}$. These two patterns show the peaks in 38.26, 44.47, 64.71,77.74, and 81.91 that could be attributed to (111), (200), (220), (311), and (222) crystallographic planes of the face-centered cubic (fcc) structure. Based on reference database ICDD/ICSD from X'Pert High Score Plus (reference code: 01-087-0719), these peaks are aspecific compound to the crystalline of Ag. The particle size has been calculated using DebyeScherrer equation (3):

$$
\mathrm{D}=\frac{k \lambda}{\beta \cos \theta}
$$

where $D$ is the average crystalline particles size of Ag-NPs, $K$ is the Scherrer constant with value of 0.9, $\lambda$ represents the X-ray wavelength (radiation/wavelength $\mathrm{Cu} K$-alpha is $0.154 \mathrm{~nm}$ ), and $\theta$ is the Bragg angle which is $2 \theta=38.30 \circ, \theta=19.15 \circ$. Here in, $\beta$ is the full width at half maximum of the diffraction peak. It can be found that the calculated average size is $33.81 \mathrm{~nm}$ which was almost the same under TEM observation.

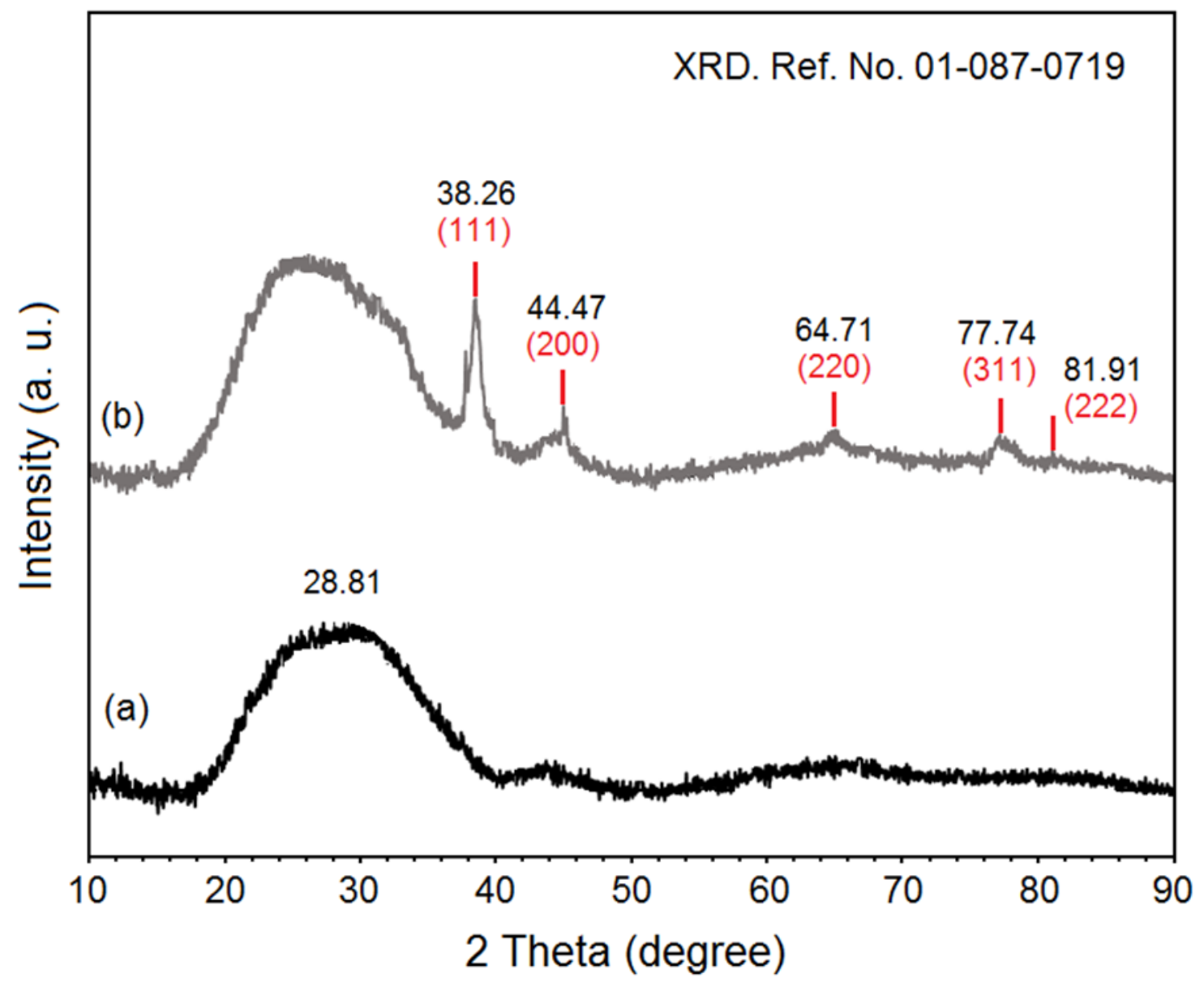

Figure 4. The XRD Pattern of J. regia (a) and J. regia/ iron oxide nanoparticles (b).

\subsection{Ultraviolet-visible Spectroscopy Analysis}

The aqueous extract of $J$. regia green husk and $\mathrm{Ag} / J$. regia was measured by UV-Vis spectroscopy over the wavelength range from $300-800 \mathrm{~nm}$ was showedin Fig. $5 \mathrm{a}$ and $\mathrm{b}$. there is no any absorption peak for aqueous extract of $J$. regia green husk (green). The surface plasmon resonance (SPR) bands 
are influenced by the size, shape, morphology, composition and dielectric environment of the prepared nanoparticles. Previous studies have shown that the spherical Ag-NPs contribute to the absorption bands at around $420-450 \mathrm{~nm}$ in the UV-visible spectra. These absorption bands were assumed to correspond to the Ag-NPs with relatively small size (less than $40 \mathrm{~nm}$ ) [31]. UV-Vis absorption spectra showed that the broad SPR band contained one peak at $430 \mathrm{~nm}$. This peak illustrates the presence of ahomogeneous distribution of hydrosol Ag-NPs after $10 \mathrm{~h}$ of stirring times and also indicates that the concentration of Ag-NPs has increased with the increase of absorbance.

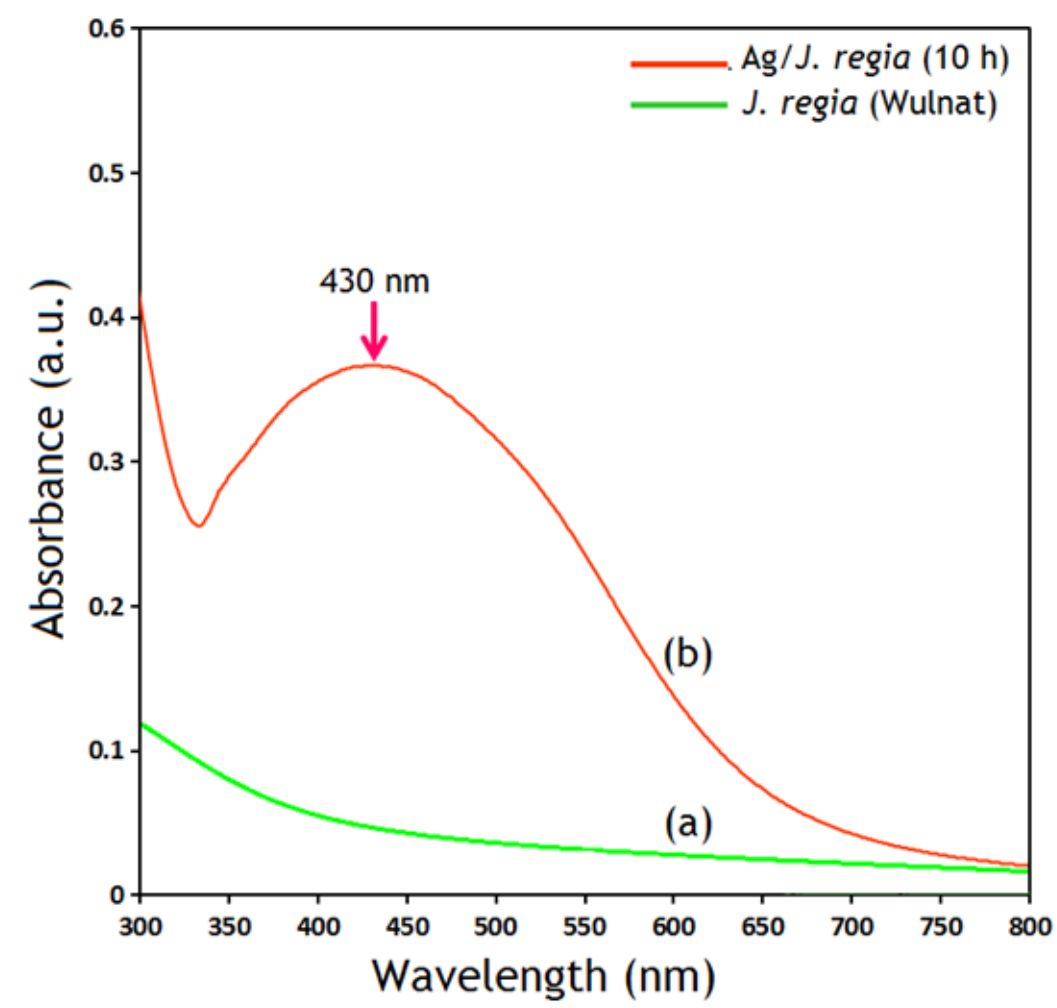

9 Figure 5. UV-vis absorption spectra of J. regia extract (a) and Synthesized Ag-NPs at room temperature (b).

\subsection{Zeta potential analysis}

The Zeta analysis was carried out to find the potential to gain information regarding the surface features of the nanoparticles. Long-term stability of particulate systems may be indicated by this equipment. A suspension that is stable for electrostatic repulsion that is physically stabilized using the value of zeta value of approximately $\pm 30 \mathrm{mV}$ is needed. Furthermore, an integrated stabilization of electrostatic and steric at $\pm 20 \mathrm{mV}$ is suitable[32]. The potential for zeta of $J$. regia contains a value of $-25.96 \mathrm{mV}$, while the values of $A g / J$. regia in room temperature transform to $29.14 \mathrm{mV}$ (Fig6a and b). According to the appropriate value for solution stability $( \pm 20 \mathrm{mV})$, the $A g / J$. regia demonstrate appropriate stability. The $A g / J$. regia in room temperature is gradually lowered, but with suitable amounts of stable expression, thus resulting in the stable $A g / J$. regia nanoparticles. 


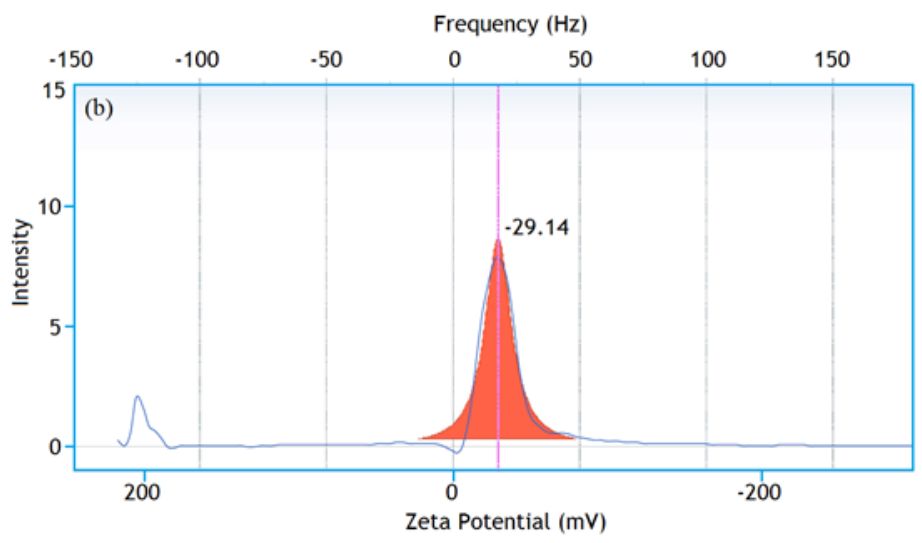

Frequency $(\mathrm{Hz})$

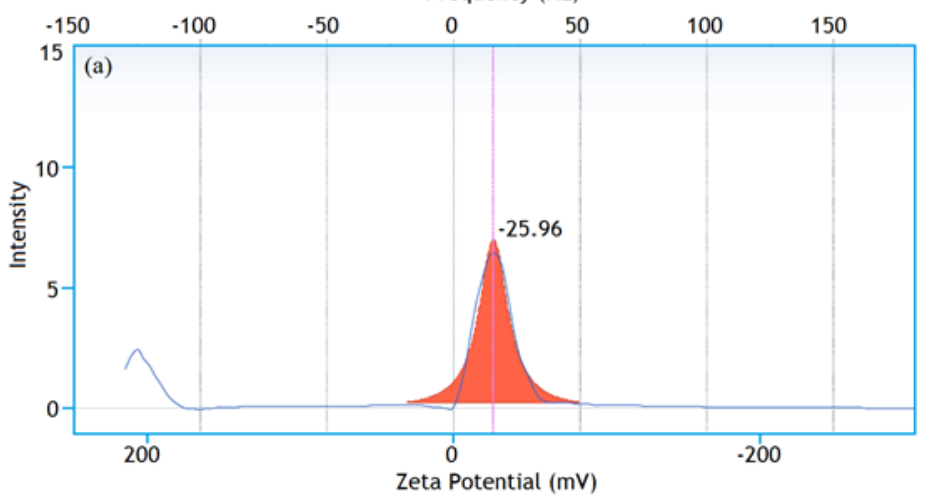

2 Figure 6. Zeta potential results for (a) J. regia and (b) Ag/J. regia at room temperature respectively.

\subsection{Morphology Study}

$4 \quad$ The image of the TEM was used to examine the shape and size of the Ag-NPs that are synthesized

5 and it reveals that many of the nanoparticles are spherical. The image shows that most of the 6 agglomerated particles are present because of the thickening traits of the green husk of J. regia[33]. In 7 addition, the act of agglomeration is expected as the Ag-NPs that are synthesized are small and 8 possess magnetic characteristics[34]. A histogram of the particle size distribution was carried out 9 based on the sizes of the 100 nanoparticles. Also, TEM result can be observed clearly that Ag-NPs 10 surrounded by the J. regia extract in the high magnification of TEM. Thus, the TEM image and their 11 size distribution are shown in Figure 7, the result showed narrow particle size distributions, with diameters in the range of $24.27-38.47 \mathrm{~nm}$. Moreover, the mean diameter and standard deviation of Ag-NPs is $31.37 \pm 7.10 \mathrm{~nm}$. 

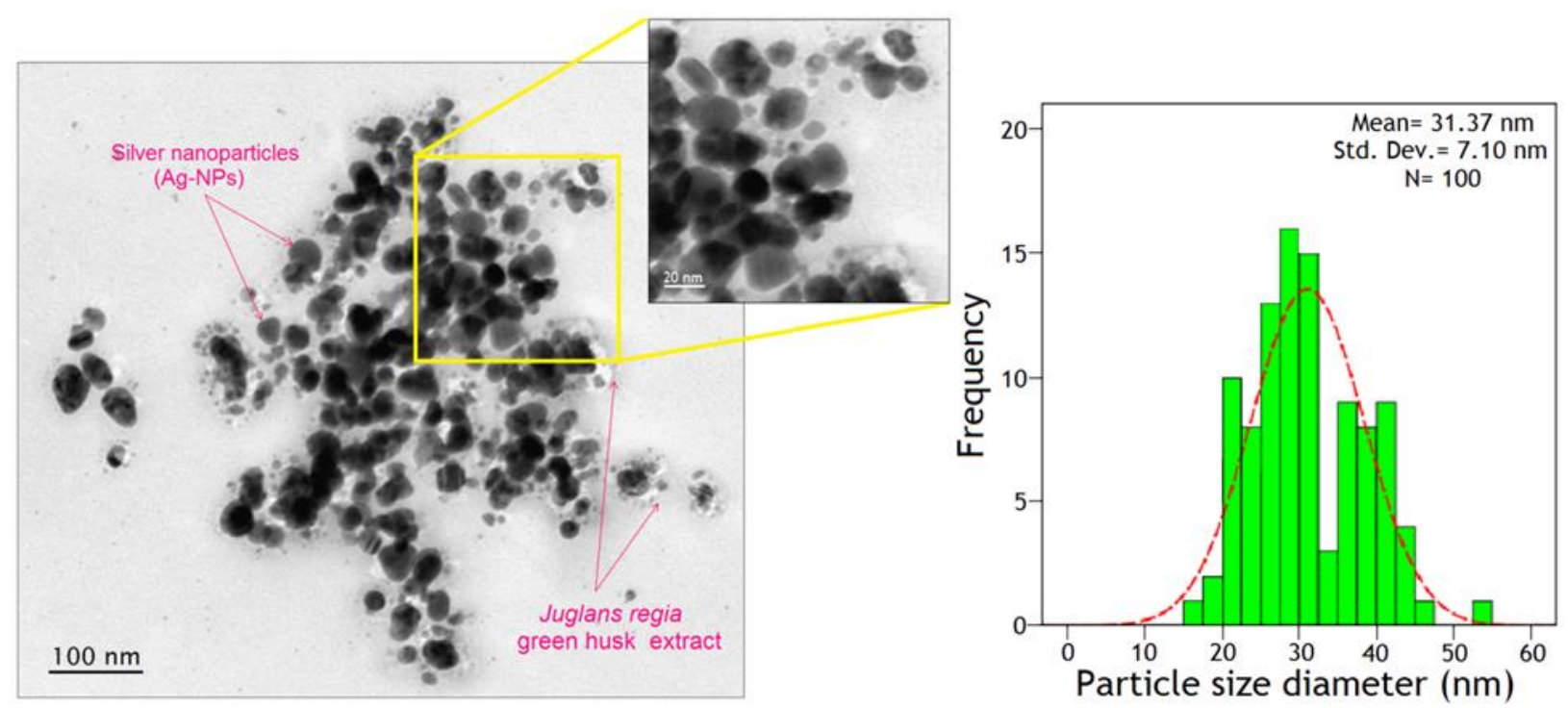

Figure 7. TEM images and corresponding size distributions of Ag/J. regia after $10 \mathrm{~h}$ from the reaction time.

The result of the AFM reveals the surface morphology of the Ag-NPs that is formulated in the media for J. regia green husk. AFM's established value and close to the established TEM, and the $J$. regia green husk films having the Ag-NPs demonstrated a densely uniformed and packed structure. Figure 8a and $\mathrm{b}$ represent the three diameters and two diameters of surface structure for Ag-NPs, while Figure $8 \mathrm{c}$ display transverse profiles depicting the looming and depth of the surface structure formed by the tapping mode. Therefore, the green husk films of Ag-NPs J. regia could offer a biocompatible and rough surface for unique applications of biology, including the immobilization of the cells.
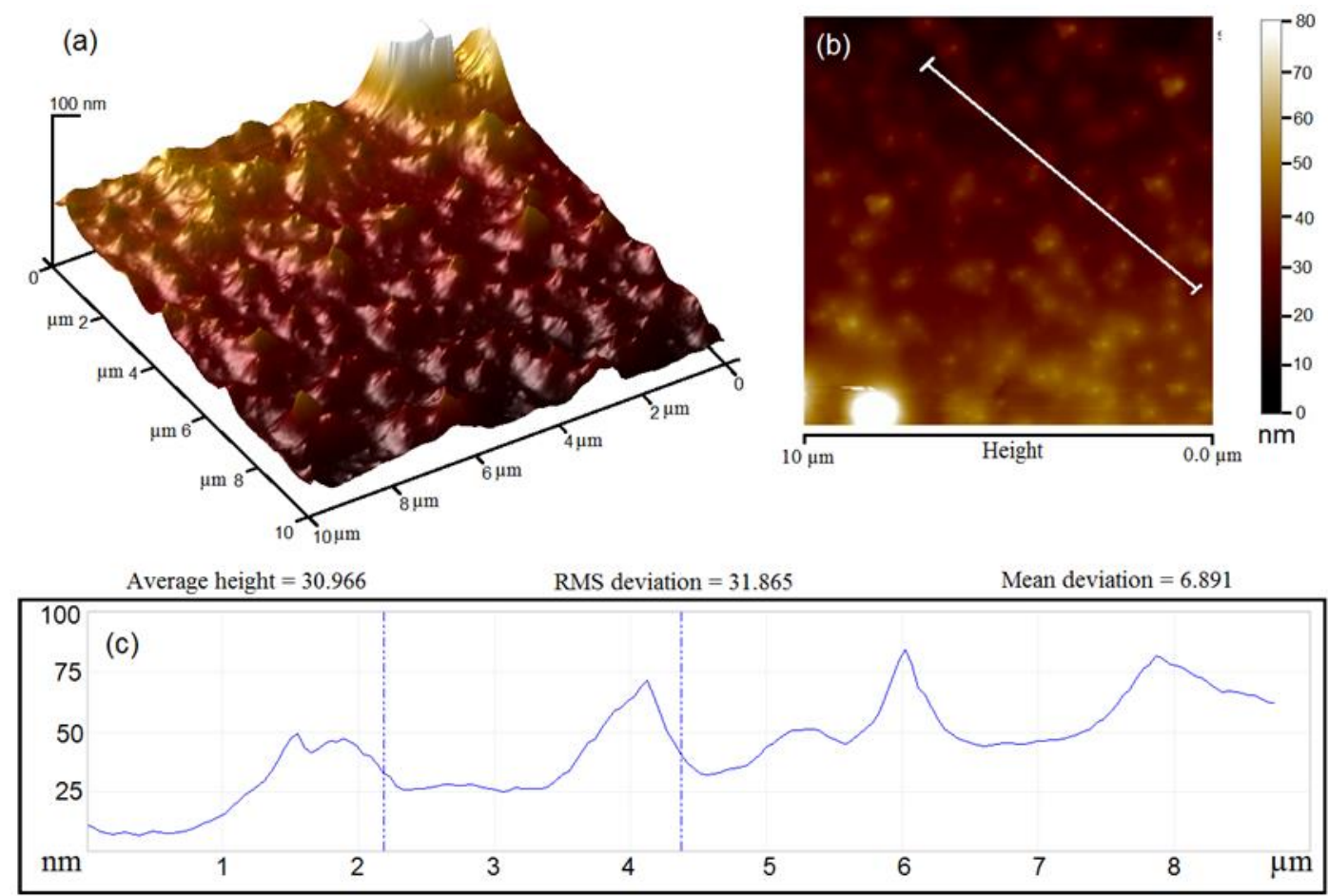

Figure 8. Atomic force microscopy images of $\mathrm{Ag} / J$. regia after $10 \mathrm{~h}$ and display transverse profiles depicted (ac). 
1

\subsection{Antimicrobial tests}

In this antibacterial study, inhibition zone values were obtained for the Ag-NPs against both Gram positive (S. aureus and B. cereus) and Gram negative (E. coli and P. aeruginosa) bacteria were examined using disc diffusion technique, and resultsare represented in Table 1. An average inhibition zone of $19( \pm 1)$ and $13( \pm 1) \mathrm{mm}$ were determined for Ag-NPs against S. aureus and B. cereus, respectively. An average inhibition zone of $9 \pm 1) \mathrm{mm}$ was measured against $E$. coli for Ag-NPs. Also, no activity was detected against the $P$. aeruginosa bacteria. The inhibition activity of antibacterial agents depends on their permeability into the ribosomes of microorganisms or microbial cells [35]. Although no significant difference was determined between the antibacterial activities of Ag-NPs, based on our observations with naked eyes, both esters had greater inhibition zones against Grampositive bacteria compared to Gram-negative ones. This is due to the fact that most Gram-negative bacteria have a thicker cell wall than Gram-positive bacteria and are thus more difficult to penetrate into their cells [36-37].

Table 1. Antibacterial inhibition zone (mm) of Ag-NPs.

\begin{tabular}{lllll}
\hline \multirow{2}{*}{ Samples } & \multicolumn{4}{l}{ Inhibition zone $\mathbf{( m m )}$} \\
\cline { 2 - 5 } & \multicolumn{2}{l}{ Gram positive bactria } & \multicolumn{2}{l}{ Gram negative bacteria } \\
\cline { 2 - 5 } & S. aureus & B. cereus & E. coli & P. aeruginosa \\
\hline Ampicillin & 44 & 35 & 26 & NA \\
J. regia & NA & NA & NA & NA \\
Ag-NPs & 19 & 13 & 9 & NA \\
\hline
\end{tabular}

$N \mathrm{~A}^{*}$ : No activity found at the tested concentration.

\section{Conclusions}

In this research, silver nanoparticles were synthesis in room temperature by using simple and biosynthesis method. According to the results, the aqueous extract of J. regia green husk has a great effect in reducing and stabilizing of Ag+ to Ag • at the room temperature. The Ag-NPs were subject to characterized by UV-vis, XRD, zeta potential, TEM and AFM. The XRD represents crystalline of Ag-NPs without apparent impurities. According to the result obtained from zeta potential analysis the Ag-NPs were improved after synthesized Ag-NPs by using plant extract. According to the result mentioned above, AFM's established value and close to the established TEM result with an average size and standard deviation of $31.37 \pm 7.1 \mathrm{~nm}$ at the room temperature. The zeta potential analysis indicated that $J$. regia green husk extract has negative charge and zeta potential value increasing by fabricate Ag-NPs. The use of J. regia green husk of the plant takes full advantage of the waste material in any desired is economically friendly, safe and efficient. Using green source as J. regia for the biosynthesis of paper Ag-NPs is a better alternative compared to the chemical or physical synthesis, since this is the free biosynthesis and ecological pollutants. Antibacterial activity of Ag-NPs was demonstrated, and showed strong antibacterial activity against Gram-positive more than Gramnegative bacteria. Needless to say, further studies are required to investigate the bactericidal effects of Ag-NPs on different types of bacteria for potential widening of this subject area, such as surgical devices or as drug delivery vehicles. 


\section{Acknowledgement}

This research was funded by Takasago Thermal Engineering Co. Ltd. grant (R.K.130000.7343.4B422) from the research management center (RMC) of Universiti Teknologi Malaysia (UTM) and Malaysia Japan International Institute of Technology (MJIIT). The authors would like to appreciate IRAJ SHAMELI, for providing walnut green husk from his garden in the Sorkh-e-Hesar Tehran (IRAN). This research and its development in nanoparticle biosynthesis and its application in medicine are inspired by his ideas.

\section{References}

[1] K. Shameli, M.B. Ahmad, E.A.J. Al-Mulla, P. Shabanzadeh, S. Bagheri, Antibacterial effect of silver nanoparticles on talc composites, Res. Chem. Intermed. 2015. 41, pp. 251-263. DOI 10.1007/s11164013-1188-y

[2] Z. Ma, J. Liu, Y. Liu, X. Zheng, K. Tang, Green synthesis of silver nanoparticles using soluble soybean polysaccharide and their application in antibacterial coatings, Int. J. Biol. Macromol. 2021. 166, pp. 567-577. DOI: 10.1016/j.ijbiomac.2020.10.214

[3] M.W. Ahmad, S. Verma, D.-J. Yang, M.U. Islam, A. Choudhury, Synthesis of silver nanoparticlesdecorated poly (m-aminophenol) nanofibers and their application in a non-enzymatic glucose biosensor, J. Macromol. Sci. A JMACROMOL SCI A. 2021. pp. 1-17. doi.org/10.1080/10601325.2021.1886585

[4] R.F. Elsupikhe, K. Shameli, M.B. Ahmad, N.A. Ibrahim, N. Zainudin, Green sonochemical synthesis of silver nanoparticles at varying concentrations of $\kappa$-carrageenan, Nanoscale Res. Lett. 2015. 10, pp. 1. DOI 10.1186/s11671-015-0916-1

[5] A.M. Narciso, C.G. da Rosa, M.R. Nunes, W.G. Sganzerla, C.M. Hansen, A.P.Z. de Melo, J.V. Paes, F.C. Bertoldi, P.L.M. Barreto, A.V. Masiero, Functionalization of bone grafts with green silver nanoparticles for biomedical application, Biocatal. Agric. Biotechnol. 2021. pp. 102074. doi.org/10.1016/j.bcab.2021.102074

[6] K. Shameli, M. Bin Ahmad, S.D. Jazayeri, S. Sedaghat, P. Shabanzadeh, H. Jahangirian, M. Mahdavi, Y. Abdollahi, Synthesis and characterization of polyethylene glycol mediated silver nanoparticles by the green method, Int. J. Mol. 2012. 13, pp. 6639-6650. doi.org/10.3390/ijms13066639

[7] Y.-y. Ren, H. Yang, T. Wang, C. Wang, Green synthesis and antimicrobial activity of monodisperse silver nanoparticles synthesized using Ginkgo Biloba leaf extract, Phys. Lett. A. 2016. 380, pp. 3773-3777. doi.org/10.1016/j.physleta.2016.09.029

[8] V.V. Mody, R. Siwale, A. Singh, H.R. Mody, Introduction to metallic nanoparticles, J. Pharm. Bioallied Sci. 2010. 2, pp. 282. doi: 10.4103/0975-7406.72127

[9] G.R. Sánchez, C.L. Castilla, N.B. Gómez, A. García, R. Marcos, E.R. Carmona, Leaf extract from the endemic plant Peumus boldus as an effective bioproduct for the green synthesis of silver nanoparticles, Mater. Lett. 2016. 183, pp. 255-260. doi.org/10.1016/j.matlet.2016.07.115

[10] N. Aihara, K. Torigoe, K. Esumi, Preparation and characterization of gold and silver nanoparticles in layered laponite suspensions, Langmuir. 1998. 14, pp. 4945-4949.

[11] X.Z. Lin, X. Teng, H. Yang, Direct synthesis of narrowly dispersed silver nanoparticles using a single-source precursor, Langmuir. 2003. 19, pp. 10081-10085. doi.org/10.1021/la035185c 
[12] A. Taleb, C. Petit, M. Pileni, Synthesis of highly monodisperse silver nanoparticles from AOT reverse micelles: a way to 2D and 3D self-organization, Chem. Mater. 1997. 9, pp. 950-959. doi.org/10.1021/cm960513y

[13] F. Raheman, S. Deshmukh, A. Ingle, A. Gade, M. Rai, Silver nanoparticles: novel antimicrobial agent synthesized from an endophytic fungus Pestalotia sp. isolated from leaves of Syzygium cumini (L), Nano Biomed Eng. 2011. 3, pp. 174-178. DOI: 10.5101/nbe.v3i3.p174-178.

[14] Ö. Dag, O. Samarskaya, N. Coombs, G.A. Ozin, The synthesis of mesostructured silica films and monoliths functionalised by noble metal nanoparticles, J. Mater. Chem. 2003. 13, pp. 328-334. https://doi.org/10.1039/B209153B

[15] R.M. Crooks, B.I. Lemon III, L. Sun, L.K. Yeung, M. Zhao, Dendrimer-encapsulated metals and semiconductors: synthesis, characterization, and applications, Dendrimers III, Springer.2001, pp. 81-135.

[16] J.L. Elechiguerra, J.L. Burt, J.R. Morones, A. Camacho-Bragado, X. Gao, H.H. Lara, M.J. Yacaman, Interaction of silver nanoparticles with J. Nanobiotechnology. 2005. 3, pp. 1. doi:10.1186/1477-3155-3-6

[17] D.I. Gittins, D. Bethell, R.J. Nichols, D.J. Schiffrin, Diode-like electron transfer across nanostructured films containing a redox ligand, J. Mater. Chem. 2000. 10, pp. 79-83. DOI: 10.1039/A902960E

[18] N.M. Huang, S. Radiman, H.N. Lim, P.S. Khiew, W.S. Chiu, K. Lee, A. Syahida, R. Hashim, C.H. Chia, $\gamma$-Ray assisted synthesis of silver nanoparticles in chitosan solution and the antibacterial properties, Chem. Eng. Sci. 2009.155, pp. 499-507. doi.org/10.1016/j.cej.2009.07.040

[19] V.K. Sharma, R.A. Yngard, Y. Lin, Silver nanoparticles: green synthesis and their antimicrobial activities, Adv. Colloid Interface Sci. 2009. 145, pp. 83-96. doi.org/10.1016/j.cis.2008.09.002

[20] M. Darroudi, M.B. Ahmad, K. Shameli, A.H. Abdullah, N.A. Ibrahim, Synthesis and characterization of UV-irradiated silver/montmorillonite nanocomposites, Solid State Sci. 2009.11, pp. 1621-1624. doi.org/10.1016/j.solidstatesciences.2009.06.016

[21] K. Shameli, M.B. Ahmad, W.M.Z.W. Yunus, A. Rustaiyan, N.A. Ibrahim, M. Zargar, Y. Abdollahi, Green synthesis of silver/montmorillonite/chitosan bionanocomposites using the UV irradiation method and evaluation of antibacterial activity, Int. J. Nanomedicine. 2010. 5, pp. 875-887. doi: $10.2147 /$ IJN.S13632

[22] K. Shameli, M.B. Ahmad, A. Zamanian, P. Sangpour, P. Shabanzadeh, Y. Abdollahi, M. Zargar, Green biosynthesis of silver nanoparticles using Curcuma longa tuber powder, Int. J. Nanomedicine. 2012. 7, pp. 5603. doi: 10.2147/IJN.S36786

[23] F. Stampar, A. Solar, M. Hudina, R. Veberic, M. Colaric, Traditional walnut liqueur-cocktail of phenolics, Food Chem. 2006. 95, pp. 627-631. doi.org/10.1016/j.foodchem.2005.01.035

[24] A. Fernández-Agulló, E. Pereira, M. Freire, P. Valentao, P. Andrade, J. González-Álvarez, J. Pereira, Influence of solvent on the antioxidant and antimicrobial properties of walnut (Juglans regia L.) green husk extracts, Ind Crops Prod. 2013. 42, pp. 126-132. doi.org/10.1016/j.indcrop.2012.05.021

[25] K. Ghasemi, Y. Ghasemi, A. Ehteshamnia, S.M. Nabavi, S.F. Nabavi, M.A. Ebrahimzadeh, F. Pourmorad, Influence of environmental factors on antioxidant activity, phenol and flavonoids contents of walnut (Juglans regia L.) green husks, J. Med. Plant Res. 2011. 5, pp. 1128-1133. 
[26] M. Carvalho, P.J. Ferreira, V.S. Mendes, R. Silva, J.A. Pereira, C. Jerónimo, B.M. Silva, Human cancer cell antiproliferative and antioxidant activities of Juglans regia L, Food Chem. Toxicol. 2010. 48, pp. 441-447. doi.org/10.1016/j.fct.2009.10.043

[27] S.N. COSMULESCU, I. Trandafir, G. Achim, B. Mihai, A. Baciu, M. Gruia, Phenolics of green husk in mature walnut fruits, Notulae Botanicae Horti Agrobotanici Cluj-Napoca 2010. 38, pp. 53. doi.org/10.15835/nbha3814624

[28] Z. Izadiyan, K. Shameli, H. Hara, S.H.M. Taib, Cytotoxicity assay of biosynthesis gold nanoparticles mediated by walnut (Juglans regia) green husk extract, J. Mol. Struct. 2018. 1151, pp. 97-105. doi.org/10.1016/j.molstruc.2017.09.039

[29] C. Singh, V. Sharma, P.K. Naik, V. KHandelwal, H. Singh, A green biogenic approach for synthesis of gold and silver nanoparticles using Zingiber officinale, Digest J. Nanomater. Biostruct. 2011. 6, pp. 535-542.

[30] L. Rabinovich-Guilatt, P. Couvreur, G. Lambert, D. Goldstein, S. Benita, C. Dubernet, Extensive surface studies help to analyse zeta potential data: the case of cationic emulsions, Chem. Phys. Lipids. 2004. 131, pp. 1-13. doi.org/10.1016/j.chemphyslip.2004.04.003

[31] N.R. Chowdhury, M. MacGregor-Ramiasa, P. Zilm, P. Majewski, K. Vasilev, 'Chocolate'silver nanoparticles: Synthesis, antibacterial activity and cytotoxicity, J. Colloid Interface Sci. 2016. 482, pp. 151-158. doi.org/10.1016/j.jcis.2016.08.003

[32] M. Şen, E.N. Erboz, Determination of critical gelation conditions of $\kappa$-carrageenan by viscosimetric and FT-IR analyses, Food Res. Int. 2010. 43. , pp. 1361-1364. doi.org/10.1016/j.foodres.2010.03.021

[33] S. Venkateswarlu, M. Yoon, Surfactant-free green synthesis of Fe $3 \mathrm{O} 4$ nanoparticles capped with 3, 4-dihydroxyphenethylcarbamodithioate: stable recyclable magnetic nanoparticles for the rapid and efficient removal of $\mathrm{Hg}$ (II) ions from water, Dalton Trans. 2015. 44, pp. 18427-18437. doi.org/10.1039/C5DT03155A

[34] M.B. Gawande, A.K. Rathi, I.D. Nogueira, R.S. Varma, P.S. Branco, Magnetite-supported sulfonic acid: a retrievable nanocatalyst for the Ritter reaction and multicomponent reactions, Green Chem. 2013. 15, pp. 1895-1899. doi.org/10.1039/C3GC40457A

[35] M.H. Soliman, G.G. Mohamed, Cr (III), Mn (II), Fe (III), Co (II), Ni (II), Cu (II) and Zn (II) new complexes of 5-aminosalicylic acid: spectroscopic, thermal characterization and biological activity studies, Spectrochim. Acta A Mol. Biomol. Spectrosc. 2013. 107, pp. 8-15. doi.org/10.1016/j.saa.2013.01.021

[36] S. Yousefi, S. Bayat, M.B.A. Rahman, Z. Ibrahim, E. Abdulmalek, Synthesis and in vitro Bioactivity Evaluation of New Galactose and Fructose Ester Derivatives of 5-Aminosalicylic Acid, CHEM BIODIVERS. 2017. 14. doi.org/10.1002/cbdv.201600362

[37] A. Smith, P. Nobmann, G. Henehan, P. Bourke, J. Dunne, Synthesis and antimicrobial evaluation of carbohydrate and polyhydroxylated non-carbohydrate fatty acid ester and ether derivatives, Carbohydr. Res. 2008. 343, pp. 2557-2566. doi.org/10.1016/j.carres.2008.07.012 